PALABRAS CLAVE

Empresas industriales

Productos manufacturados

Industria automotriz

Industria electrónica

Competitividad

Capacitación de la mano de obra

Modelos econométricos

Estudios de casos

México

Ramón Padilla

Oficial de Asuntos Económicos

œramon.padilla@cepal.org

Miriam Juárez

Economista

œMijt@hotmail.com

Unidad de Comercio

Internacional e Industria,

CEPAL, Sede Subregional en México
REVISTA DE LA CEPAL 92 • AGOSTO 2007

\section{Efectos de la capacitación en la competitividad de la industria manufacturera}

\author{
Ramón Padilla y Miriam Juárez
}

$\mathrm{E}$

ste artículo examina el efecto de la capacitación en la competitividad de la industria manufacturera, distinguiendo entre ramas industriales con distintas características tecnológicas y productivas. Aplicando un enfoque sistémico, además de estudiar las actividades dentro de las empresas y el impacto de la capacitación en ellas, analiza el entorno organizacional e institucional de apoyo a la capacitación y el efecto de esta última en la localidad en su conjunto. El análisis considera dos niveles: a nivel de empresa (análisis micro) estudia la industria manufacturera en México haciendo uso de herramientas econométricas, y a nivel de región (análisis meso) estudia la industria electrónica en una región de México. La evidencia empírica permite concluir que la capacitación en la empresa tiene un impacto diferenciado en la competitividad de industrias con distintas características tecnológicas y tiene también un impacto positivo en la región a través de la difusión de conocimientos. 
I

\section{Introducción}

La competitividad a distintos niveles (empresa, región, industria o país) ha tomado un papel central en la agenda de desarrollo. En los países latinoamericanos, caracterizados por economías cada vez más abiertas e integradas a cadenas globales de producción, el mejoramiento de la competitividad es fundamental para la consecución de mayores niveles de desarrollo económico y social. En efecto, la competitividad está asociada con la capacidad de participar exitosamente en mercados internacionales, la generación de valor agregado y la creación de empleo, entre otros factores.

La competitividad puede adoptar diversas formas. Puede estar basada en ventajas competitivas estáticas, como recursos naturales abundantes o bajos costos salariales. Pero también puede estar sustentada en ventajas comparativas dinámicas, producto de introducir nuevos y mejores productos, implementar nuevas formas de organización empresarial o incrementar la capacidad productiva (McFetridge, 1995; Spencer y Hazard, 1988; Porter, 1985). La inversión en capital humano es un elemento central para la creación y fortalecimiento de ventajas comparativas dinámicas, las cuales son sostenibles y ofrecen un gran potencial de desarrollo económico y social.

En el contexto actual, dominado por el constante y rápido cambio tecnológico, la capacitación en la empresa - como una forma de creación del capital humano - tiene un papel muy importante en el fortalecimiento de la competitividad. Por un lado, complementa la educación formal porque ofrece al trabajador los conocimientos y habilidades necesarios para hacer uso de tecnología, adaptarla y eventualmente mejorarla (Booth y Snower, 1996). Por otro lado, al estar dirigida a proveer los conocimientos y habilidades que los empleados necesitan para sus actividades diarias, cabe esperar que signifique rápidos y significativos rendimientos para la empresa (Tan y Batra, 1995; Mincer, 1994).

Este trabajo pretende examinar el impacto de la capacitación dentro de la empresa en la competitividad

$\square$ Los autores agradecen a Claudia Schatan y Juan Carlos Moreno sus comentarios a los primeros borradores de este trabajo. También agradecen a un evaluador anónimo su valiosa retroalimentación. de tres ramas de la industria manufacturera con distintas características productivas y tecnológicas. Para ello propone un enfoque sistémico, es decir, además de estudiar las actividades al interior de las empresas y el efecto de la capacitación en ellas, analiza el entorno organizacional e institucional de apoyo a la capacitación, así como el impacto que esta tiene en la localidad. El análisis se efectúa a nivel de empresa (micro) y a nivel regional (meso). Este enfoque permite identificar el impacto de la capacitación no solo en la competitividad de la empresa, sino también en la competitividad de su entorno geográfico, reconociendo que, además de los beneficios privados que obtienen las empresas, la capacitación origina también un beneficio social.

La identificación del componente micro se fundamenta en el análisis estadístico y econométrico de una base de datos pública, la Encuesta Nacional de Empleo, Salarios, Tecnología y Capacitación (ENESTYC) 2001, de México. A diferencia de otros estudios empíricos que han hecho uso de esta encuesta, la presente investigación considera la hipótesis de que la capacitación tiene un impacto diferenciado en la competitividad de distintas industrias. El análisis del componente meso (regional) se basa en trabajo de campo realizado en México en octubre de 2005. Debido al impacto diferenciado en la competitividad y a las características que la capacitación en la empresa tiene en distintas ramas industriales, se seleccionó una industria para efectuar este análisis meso. La industria seleccionada es la electrónica, principalmente por su mayor propensión a capacitar y por exhibir el mayor impacto relativo de la capacitación en la competitividad entre las tres ramas consideradas en esta investigación.

El resto del artículo se divide en cuatro secciones. La sección II plantea los conceptos centrales y hace un breve repaso de estudios previos sobre el tema. La sección III presenta el componente micro, el cual, como se comentó, contiene un análisis estadístico y econométrico. La sección IV desarrolla el componente meso, y la sección $\mathrm{V}$ contiene conclusiones y recomendaciones de política. 


\section{II}

\section{Competitividad e inversión en capital humano}

Existe consenso entre organizaciones e investigadores de que el fortalecimiento de la competitividad de las empresas es un elemento esencial para la consecución de mayores niveles de desarrollo económico y social. La competitividad puede darse a distintos niveles de agregación: en la empresa, en la industria o un grupo de industrias, en la región y en el país.

La competitividad a nivel de la empresa tiene especial relevancia en este estudio y se entiende como la habilidad de esta para operar rentablemente en un mercado determinado (McFetridge, 1995). La competitividad puede adoptar formas diversas. En primer lugar, en la literatura especializada es comúnmente reconocida la diferencia entre competitividad efímera, artificial o espuria y competitividad real o auténtica. La primera está asociada con bajos salarios, explotación no sustentable de recursos naturales, condiciones laborales inadecuadas, etc. La competitividad real, en cambio, se basa en la capacidad de introducir nuevos y mejores productos, implementar nuevas formas de organización empresarial e incrementar la capacidad productiva, entre otras cosas (Spencer y Hazard, 1988).

Del párrafo anterior se desprende cuán complejo es contar con un único indicador comúnmente aceptado para medir o estimar la competitividad. Sin embargo, es posible identificar un conjunto de factores frecuentemente usados para este propósito: rentabilidad, productividad, costos, valor agregado, participación de mercado, exportaciones, innovación tecnológica y calidad de los productos, entre otros.

Como se mencionó, la competitividad también puede ser entendida a nivel de industria, región o país. A nivel meso o macro, la competitividad está asociada a ventajas comparativas derivadas de los recursos disponibles en la región o el país, ya sean recursos naturales o fuerza de trabajo abundantes, o ventajas creadas a través de inversión en capital humano, infraestructura o capacidades tecnológicas (BID, 2004). Un análisis agregado más integral lo ofrece el concepto de competitividad sistémica. ${ }^{1}$

En la competitividad a nivel de empresa influyen muchos factores, entre otros los siguientes: un entorno

\footnotetext{
${ }^{1}$ Véase Altenburg, Hillebrand y Meyer-Stamer (1998).
}

macroeconómico estable; un sistema financiero sólido; la habilidad para usar, adaptar y crear nuevas tecnologías; la habilidad para atraer, formar y retener capital humano. ${ }^{2}$ Este último es el objeto central de la presente investigación.

El capital humano, producto de la educación formal y el aprendizaje posterior a esta, ha sido reconocido ampliamente en la teoría económica como un factor central para el desarrollo económico (Romer, 1989; Mincer, 1981; Becker, 1964). En el contexto actual de una economía globalizada y dominada por el constante y rápido cambio tecnológico, donde el conocimiento es considerado uno de los principales factores determinantes de la competitividad, el capital humano es fundamental para elevar la productividad y alcanzar mayores niveles de bienestar (Tan y Batra, 1995). La adquisición de conocimientos y habilidades permite a los trabajadores adaptarse más fácilmente a nuevas exigencias en sus puestos de trabajo. Para que una empresa mantenga su competitividad en un entorno de continuos cambios en preferencias y tecnologías, se necesitan trabajadores capaces de cambiar e innovar rápidamente (Booth y Snower, 1996).

La capacitación en la empresa es uno de los componentes principales de la inversión de un país en capital humano. En algunos países de ingreso medio y alto inclusive compite en términos de importancia con la inversión en educación formal (Tan y Batra, 1995; Mincer, 1994). La capacitación en la empresa es entendida como el conjunto de actividades formales e informales que buscan la transmisión de conocimientos y/o el desarrollo de habilidades en los empleados. Por lo tanto, es un concepto más amplio que la capacitación en el trabajo, la cual está asociada solo con actividades informales de transmisión de conocimientos, a través de la demostración y la práctica. ${ }^{3}$ Es importante también reconocer que la capacitación en la empresa puede incluir dos aspectos: la capacitación general aplicable en más de una empresa, y la capacitación específica relacionada con conceptos y habilidades propias de una empresa. Las empresas, por la dificultad de apropiación

\footnotetext{
${ }^{2}$ Véase Nabi y Luthria (2002).

${ }^{3}$ Para más información sobre la capacitación en el trabajo, véase Lara Rivero y Díaz-Berrio (2003).
} 
de los resultados de la formación, tienden a invertir en esta última (Gallart, 2001).

Los estudios empíricos previos no han abordado de manera directa el impacto de la capacitación en la empresa sobre la competitividad. Sin embargo, diversos estudios empíricos, haciendo uso de herramientas econométricas, han mostrado que dicha capacitación tiene un efecto positivo y significativo en la productividad total de los factores (Tan y López-Acevedo, 2003; Tan y Batra, 1995; Bartel, 1989). La evidencia empírica también indica que la probabilidad de que un empleador ofrezca capacitación está asociada con distintas variables, como el tamaño de la empresa, el nivel educativo de los empleados, las inversiones en nuevas tecnologías, la orientación exportadora, el uso de métodos de control de calidad y la presencia de capital extranjero. ${ }^{4}$

\section{III}

\section{Capacitación y competitividad a nivel micro}

Los objetivos de esta sección son estudiar, haciendo uso de técnicas econométricas, cuáles son las variables que están altamente asociadas con el desempeño positivo de la competitividad de la empresa, y analizar el impacto de la capacitación en la competitividad de la empresa.

La competitividad es un concepto complejo y no hay consenso sobre un indicador cuantitativo único para su estimación. En la literatura económica a menudo se usa la productividad como el mejor indicador para estimar la competitividad. En términos generales, la productividad se define como la tasa de un volumen de medida del producto con relación a un volumen de medida del uso de factores productivos. En este sentido se puede hablar de la productividad de la mano de obra, la productividad del capital o la productividad total de los factores. La productividad es una buena variable representativa de competitividad porque incluye muchos aspectos de la competitividad de una empresa, región o país, y su estimación es factible. ${ }^{5}$

En estudios empíricos previos se ha analizado el impacto de la capacitación sobre la competitividad en el agregado de la industria manufacturera. Sin embargo, este estudio parte del hecho de que las necesidades de adquirir nuevos conocimientos y difundirlos entre el personal, así como la fuente principal de dichos conocimientos, no son homogéneos en todas las ramas de la industria manufacturera, como tampoco lo son los factores que determinan la competitividad. En efecto, las industrias que hacen uso intensivo de tecnología adquieren nuevos conocimientos principalmente a través de actividades internas de diseño e investigación y desarrollo (I+D),

\footnotetext{
${ }^{4}$ Véase Tan y López-Acevedo (2003); Batra y Tan (2002); Booth y Snower (1996); Lynch y Black (1995); Tan y Batra (1995).

${ }^{5}$ Véase OCDE (2001).
}

mientras que las industrias manufactureras con bajo contenido tecnológico acuden a fuentes externas, como proveedores de equipo y empresas de consultoría, para adquirir nuevos conocimientos. ${ }^{6}$

Con esta distinción en mente, esta sección explora las siguientes preguntas: i) ¿la capacitación tiene un impacto diferenciado en la competitividad de industrias con distintas características tecnológicas?; ii) en caso afirmativo, ¿cuáles son los rasgos distintivos de la capacitación en distintas industrias?, y iii) ¿cuáles son las variables asociadas con un desempeño favorable de la competitividad en industrias con distintas características tecnológicas?

Para contestar las preguntas anteriores se seleccionaron tres ramas industriales ${ }^{7}$ con distintas características productivas y tecnológicas: la de confección de prendas de vestir, la automotriz y la electrónica. ${ }^{8}$ Para el análisis estadístico y econométrico se utilizó la ENESTYC del año 2001, la última disponible cuando se llevó a cabo esta investigación. La ENESTYC, además de información detallada sobre las actividades de capacitación en la empresa, contiene información sobre variables asociadas con la competitividad y sus factores determinantes. La unidad de estudio de esta encuesta es el establecimiento manufacturero y tiene representatividad a nivel nacional por rama y tamaño de la planta.

\footnotetext{
${ }^{6}$ Véase la contribución seminal de Pavitt (1984), así como los trabajos de Giuliani, Pietrobelli y Rabelotti (2005) y de Cohen, Goto y otros (2002).

${ }^{7}$ Según la Clasificación Internacional Industrial Uniforme de todas las actividades económicas (CIIU/Rev. 2).

${ }^{8}$ Véase una descripción de las características productivas y tecnológicas de la confección en OCDE (2004a); de la industria automotriz en Abdel (2004), y de la industria electrónica en Padilla (2005).
} 


\section{Estadísticas descriptivas}

Las tres ramas seleccionadas para el análisis fueron: la 3220, que corresponde a confección de prendas de vestir; la 3832, que agrupa las actividades de fabricación o ensamble de equipo electrónico de radio, televisión y comunicaciones; y la 3841, que corresponde a la industria automotriz. Estas ramas, como se describe a continuación, tienen distintas características productivas y tecnológicas, lo cual, de acuerdo con la hipótesis aquí planteada, está asociado con un impacto diferenciado de la capacitación en la competitividad.

La confección de prendas de vestir es el eslabón final de una amplia cadena de valor, hace uso intensivo de mano de obra y emplea tecnología madura (OCDE, 2004a). La industria automotriz es intensiva en escala, tiene altos estándares de calidad y hace uso mediano de tecnología (Abdel, 2004). Por su parte, la industria electrónica se caracteriza por un elevado dinamismo tecnológico, altos estándares de calidad y alta eficiencia productiva (Padilla, 2005).

El cuadro 1 presenta algunos indicadores sobre las tres ramas estudiadas, calculados a partir de la ENESTYC. En el año 2000, la rama de confección abarcaba 24.084 empresas, la gran mayoría microempresas (91\%), y el valor agregado medio por planta era relativamente bajo (773.000 pesos). La rama electrónica incluía 397 empre- sas, $71 \%$ de ellas de tamaño micro, y el valor agregado medio por empresa era de 15,8 millones de pesos. Por último, la rama automotriz agrupaba a 1.370 empresas, $58 \%$ de ellas microempresas, y el valor agregado medio por planta era de 79.586 millones de pesos.

De las tres ramas examinadas, la electrónica fue la que más adquirió, usó y generó tecnologías. Así lo señalan algunos indicadores, como el mayor porcentaje de ingresos destinados a compra y transferencia de tecnología; el mayor uso de máquinas de control automatizado y robots, y el mayor porcentaje de empresas que realizan $\mathrm{I}+\mathrm{D}$. Por el contrario, la rama de la confección fue la que exhibió menor intensidad tecnológica. No obstante, es importante señalar que en México el gasto en I+D en las tres ramas es significativamente inferior al registrado por estas mismas en países desarrollados, ${ }^{9}$ que la innovación es principalmente de proceso y que la mayor parte de las innovaciones constituyen novedad en el mercado local y no en el ámbito mundial (CONACYT, 2003). En cuanto a la tecnología de organización de la producción, más del 75\% de las empresas electrónicas y automotrices cuenta con programas de control de

\footnotetext{
${ }^{9}$ Véase OCDE (2004b).
}

CUADRO 1 México: algunos indicadores de las ramas de confección
de prendas de vestir, electrónica y automotriz, 2000

\begin{tabular}{|c|c|c|c|}
\hline Indicador & $\begin{array}{l}\text { Rama de la confección } \\
(3220)\end{array}$ & $\begin{array}{l}\text { Rama electrónica } \\
\text { (3832) }\end{array}$ & $\begin{array}{l}\text { Rama automotriz } \\
(3841)\end{array}$ \\
\hline Número de empresas & 24084 & 397 & 1370 \\
\hline Valor agregado (promedio, miles de pesos) & 773,0 & 15838,6 & 79586,2 \\
\hline \multicolumn{4}{|c|}{ Adquisición, uso y generación de tecnología } \\
\hline Porcentaje de los ingresos destinado a la compra y transferencia de tecnología & 1,5 & 3,6 & 1,8 \\
\hline $\begin{array}{l}\text { Porcentaje del valor de la maquinaria y equipo en operación que son de } \\
\text { control numérico automatizado }\end{array}$ & 6,5 & 23,9 & 12,3 \\
\hline Porcentaje del valor de la maquinaria y equipo en operación que son robots & 0,1 & 9,9 & 4,9 \\
\hline Porcentaje de establecimientos que realizan I+D & 3,5 & 25,7 & 22,8 \\
\hline Porcentaje de establecimientos con sistemas de control de calidad & 53,6 & 76,8 & 76,7 \\
\hline \multicolumn{4}{|c|}{ Capacitación y recursos humanos } \\
\hline Porcentaje de empresas que capacitaron a sus trabajadores & 61,8 & 89,1 & 88,0 \\
\hline $\begin{array}{l}\text { Porcentaje de trabajadores con educación profesional (como proporción } \\
\text { de la plantilla laboral) }\end{array}$ & 5,0 & 14,2 & 12,0 \\
\hline $\begin{array}{l}\text { Porcentaje de trabajadores con educación de posgrado (como proporción } \\
\text { de la plantilla laboral) }\end{array}$ & 0,4 & 1,4 & 0,8 \\
\hline
\end{tabular}

Fuente: elaboración propia con datos de la Encuesta Nacional de Empleo, Salarios, Tecnología y Capacitación en el Sector (ENESTYC) del año 2001. 


\begin{tabular}{|c|c|c|c|c|}
\hline & $\mathrm{dF} / \mathrm{dx}$ & $\begin{array}{c}\text { Errores estimados } \\
\text { robustos }\end{array}$ & $\mathrm{Z}$ & $\mathrm{P}>|\mathrm{z}|$ \\
\hline Variable dicotómica de la rama automotriz & $-0,509$ & 0,087 & $-3,58$ & 0,000 \\
\hline Variable dicotómica de la rama de la confección & $-0,591$ & 0,114 & $-4,15$ & 0,000 \\
\hline Variable dicotómica de la adquisición de maquinaria y equipo & 0,310 & 0,097 & 3,04 & 0,002 \\
\hline Tamaño & 0,211 & 0,081 & 2,59 & 0,010 \\
\hline Nivel educativo medio & 0,038 & 0,017 & 2,12 & 0,034 \\
\hline Variable dicotómica de sindicato & 0,336 & 0,115 & 2,76 & 0,006 \\
\hline Variable dicotómica de subcontratación & 0,384 & 0,137 & 2,49 & 0,013 \\
\hline Variable dicotómica de calidad & 0,228 & 0,130 & 1,72 & 0,085 \\
\hline Observaciones & 3971 & & & \\
\hline Prob $>\mathrm{chi}^{2}$ & 0,00 & & & \\
\hline Seudo $\mathrm{R}^{2}$ & 0,47 & & & \\
\hline
\end{tabular}

Fuente: elaboración propia con datos de ENESTYC (2001).

calidad, mientras que en la rama de la confección sólo el $53 \%$ lo tiene (cuadro 1).

En cuanto a capacitación, la rama electrónica tiene la mayor proporción de empresas que capacitan a sus trabajadores (89\%), seguida por la automotriz (88\%) y por la de confección (62\%). Las empresas de la rama automotriz presentaron el mayor número de horas de capacitación por obrero al año y recurrieron en mayor proporción a agentes externos para llevarla a cabo. Por otro lado, la rama electrónica registró el mayor porcentaje medio de empleados con educación profesional y de posgrado ( $14,2 \%$ y $1,4 \%$, respectivamente) y la automotriz exhibió proporciones semejantes $(12 \%$ y $0,8 \%$, respectivamente), pero en la rama de confección los porcentajes fueron menores ( $5 \%$ y $0,4 \%$, respectivamente).

\section{El modelo econométrico}

Para estimar la productividad multifactorial se utilizó un índice calculado como el cociente del valor agregado generado y el monto del gasto en los insumos de capital y trabajo. ${ }^{10}$ La productividad multifactorial así calculada refleja las economías de escala, la eficiencia productiva y las diferencias de capacidad instalada entre las empresas. ${ }^{11}$

\footnotetext{
${ }^{10}$ Véase más información sobre el cálculo de índices de productividad en OCDE (2001).

${ }^{11}$ La variable más usada en la literatura económica para estimar la productividad multifactorial se deriva de los residuos de la estimación de una función de producción tipo Cobb-Douglas con rendimientos constantes. Sin embargo, diversos autores han identificado problemas de autoselección y simultaneidad en la estimación de la función de producción. Véase Pavcnik (2002); Olley y Pakes (1996), y Griliches (1967).
}

En primera instancia, para mostrar si efectivamente los factores asociados con las decisiones de capacitación de las empresas varían de una rama a otra, se estimó para el conjunto de las tres ramas un modelo probit de los factores determinantes de que una empresa capacite a su personal. En este modelo la variable dependiente es dicotómica: 1 si la empresa capacita y 0 si no capacita; los resultados de la estimación se presentan en el cuadro 2. Para la estimación de este modelo se partió de un conjunto de variables que incluyó el tamaño de los establecimientos, la educación de los trabajadores, la existencia de sindicatos, la presencia de capital extranjero, la actividad exportadora, la participación en actividades de subcontratación, control de calidad y variables de uso, adquisición y generación de tecnología (véase el apéndice A).

Los coeficientes señalan efectos diferenciados en la probabilidad de capacitar al personal entre las tres ramas. Las variables dicotómicas, significativas al 99\% de confianza y negativas, indican que los establecimientos pertenecientes a la rama automotriz y la de confección tienen una menor propensión a capacitar a sus trabajadores que la rama electrónica. Este resultado depende fuertemente de diferencias en la dinámica tecnológica de las tres ramas estudiadas. La electrónica y en menor medida la automotriz se caracterizan por un rápido cambio tecnológico tanto en los procesos de producción como en los productos, lo que exige personal capacitado para operar en esquemas flexibles y de cambio continuo. Otras variables asociadas significativa y positivamente con un aumento en la probabilidad de que las empresas capaciten a sus trabajadores son: la introducción de maquinaria y equipo, el nivel educativo medio de los trabajadores, el tamaño de los establecimientos y la 
existencia de sindicato. Estos hallazgos son consistentes con la evidencia empírica previa.

En seguida se estudia el efecto de la capacitación de los trabajadores sobre la competitividad y otras variables asociadas a ella, así como los rasgos distintivos de la capacitación, todo esto considerando que se trata de industrias con características tecnológicas y productivas distintas. La capacitación no puede ser tratada como una variable exógena, porque la decisión de capacitar puede estar basada en el conocimiento previo de la empresa respecto a su nivel de productividad (autoselección); por lo tanto, se propone un modelo en tres etapas para estimar el impacto de la capacitación en la competitividad (productividad como variable sustitutiva); este modelo controla los efectos de variables no observadas y la endogeneidad de la variable de tratamiento (capacitación), ${ }^{12} \mathrm{de}$ acuerdo con el procedimiento desarrollado por Barnow, Cain y Goldberger (1981). Primero, se estima un modelo de selección empleando una regresión probit. Segundo, se usan las probabilidades estimadas de la primera etapa para calcular la variable de sesgo de selección, usando el ajuste Heckman-Maddala-Lee. Tercero, se usa el método de variables instrumentales para ajustar la correlación entre los residuos del modelo probit y los residuos de la segunda etapa, con lo cual los estimadores y errores estándares calculados son consistentes. ${ }^{13} \mathrm{El}$ modelo por estimar es el siguiente:

$$
\mathrm{P}_{\mathrm{i}}=\mathbf{B}^{\prime} \mathbf{X}_{\mathbf{i}}+\delta \mathrm{C}_{\mathrm{i}}+\gamma \mathrm{HML}_{\mathrm{i}}+\mathbf{e}_{\mathbf{i}}
$$

Esta estimación captura el efecto $B$ de un conjunto de variables exógenas $\mathrm{Xi}$ sobre $\mathrm{P}_{\mathrm{i}} ; \gamma$ captura el efecto del sesgo de autoselección (HML) en la productividad $\mathrm{P}_{\mathrm{i}} ; \mathrm{C}_{\mathrm{i}}$ captura el efecto de una variable endógena binaria que indica si las empresas capacitaron o no a sus trabajadores sobre una variable de productividad $\mathrm{P}_{\mathrm{i}}$. Esta variable $\mathrm{C}_{\mathrm{i}}$ se modela como el resultado de una variable latente no observable $\mathrm{C}_{\mathrm{i}} *{ }^{14}$

\footnotetext{
${ }^{12}$ La capacitación no puede ser tratada como una variable exógena si la decisión de una empresa de capacitar está basada en el conocimiento previo de la empresa sobre el nivel de productividad, debido a un sesgo por autoselección. En este caso, las estimaciones resultantes de mínimos cuadrados ordinarios serían sesgadas e inconsistentes.

${ }^{13}$ Esta técnica emplea métodos de máxima verosimilitud, la cual maximiza la densidad conjunta de las variables dependientes observadas, con la finalidad de obtener estimadores y errores estándares consistentes. En contraste, los estimadores resultantes de una regresión de mínimos cuadrados ordinarios serían sesgados e inconsistentes. Dadas las propiedades asintóticas de los estimadores de máxima verosimilitud, el número de observaciones de la muestra aquí empleada permite obtener un buen ajuste.

${ }^{14} \mathrm{C}_{\mathrm{i}}{ }^{*}=\alpha \mathrm{W}_{\mathrm{i}}+\mathrm{u}_{\mathrm{i}}, \mathrm{C}=1 \mathrm{si}_{\mathrm{i}}^{*}>0$ ó $\mathrm{C}=0$ si $_{\mathrm{i}}{ }^{*}<0$; donde $\mathrm{C}_{\mathrm{i}}^{*}$ es el beneficio (o pérdida) neta de la capacitación y, aunque no es observable,
}

En la primera etapa — la estimación de modelos probit- la especificación para las tres ramas partió de un conjunto de variables independientes, entre otras las siguientes: educación previa de los trabajadores, tamaño de la planta, indicadores de adquisición y uso de tecnología, existencia de controles de calidad, origen del capital extranjero, actividades de subcontratación y la realización de actividades conjuntas con otras empresas. ${ }^{15}$ Las estimaciones partieron de este conjunto de variables y se fueron ajustando hasta llegar a una estimación consistente. En la segunda etapa, que corresponde a la estimación de factores relacionados con la productividad de las empresas, se introdujeron las variables de la etapa anterior, junto con otras variables correspondientes a las distintas modalidades utilizadas para impartir la capacitación ${ }^{16}$ y las categorías ocupacionales en las que se concentró la capacitación (véase el apéndice A). En esta etapa se siguió la misma metodología de eliminación de variables hasta obtener una estimación consistente.

\section{a) Rama electrónica (3832)}

El cuadro 3 muestra los resultados de la primera etapa de estimación del modelo probit de la rama electrónica. En primer lugar, el gasto en I+D como porcentaje del valor agregado se asocia positivamente con la probabilidad de que una empresa capacite a sus empleados. Esto se debe a que la introducción de nuevas tecnologías, y más aún la realización de actividades que hacen uso intensivo de conocimientos, exigen una estrategia activa de capacitación. La adquisición de maquinaria y equipo como porcentaje del valor agregado está también relacionada positivamente con la probabilidad de que las empresas capaciten. De igual forma, la compra de bienes de capital, que incorporan nueva tecnología, obliga a capacitar para su uso.

La variable de control de calidad también está asociada positivamente con la probabilidad de que los establecimientos capaciten a sus trabajadores. Esto puede explicarse porque el aseguramiento de la calidad, apoyado en sistemas modernos de administración como "control total de calidad" y "seis sigma", requiere de una política activa de capacitación de los empleados. Por otro lado, la antigüedad del establecimiento se asocia positivamente con la probabilidad de que una empresa capacite a sus trabajadores. Este resultado podría indicar

conocemos sus determinantes. $\mathrm{W}_{\mathrm{i}}$ es un vector de características para la empresa $i$ que afectan los costos y beneficios asociados a la elección de capacitar; y $u_{i}$ es el término aleatorio para la empresa $i$.

15 Véase en el apéndice A la lista completa de variables utilizadas.

${ }^{16}$ Capacitación interna frente a capacitación externa y capacitación formal frente a capacitación informal. 
México: rama electrónica (3832)

(Modelo probit de factores determinantes de la capacitación en las empresas)

\begin{tabular}{|c|c|c|c|c|}
\hline & Coeficiente & Errores estimados robustos & $\mathrm{Z}$ & $\mathrm{P}>|\mathrm{z}|$ \\
\hline Investigación y desarrollo & 4,855 & 1,605 & 3,03 & 0,002 \\
\hline Adquisición de maquinaria y equipo & 3,738 & 1,036 & 3,61 & 0,000 \\
\hline Variable dicotómica de calidad & 1,669 & 0,974 & 1,71 & 0,087 \\
\hline Capital extranjero & 0,098 & 0,069 & 1,43 & 0,152 \\
\hline Antigüedad & 0,209 & 0,043 & 4,87 & 0,000 \\
\hline Índice de actividades conjuntas & 14,163 & 3,598 & 3,94 & 0,000 \\
\hline Variable dicotómica de sindicato & 1,533 & 0,597 & 2,56 & 0,010 \\
\hline Constante & $-5,377$ & 1,282 & $-4,19$ & 0,000 \\
\hline Observaciones & 394 & & & \\
\hline Prob $>$ chi $^{2}$ & 0,00 & & & \\
\hline Seudo $\mathrm{R}^{2}$ & 0,79 & & & \\
\hline
\end{tabular}

Fuente: elaboración propia con datos de ENESTYC, 2001.

CUADRO 4

México: rama electrónica (3832)

(Método de variables instrumentales)

\begin{tabular}{|c|c|c|c|c|}
\hline & Coeficiente & Errores estimados robustos & $\mathrm{Z}$ & $\mathrm{P}>|\mathrm{z}|$ \\
\hline Variable dicotómica de capacitación & 4,867 & 2,488 & 1,96 & 0,056 \\
\hline Ajuste Heckman-Maddala-Lee & $-3,827$ & 2,089 & $-1,83$ & 0,073 \\
\hline Variable dicotómica de I+D & 1,862 & 0,746 & 2,5 & 0,016 \\
\hline Ingeniería básica & $-0,199$ & 0,060 & $-3,28$ & 0,002 \\
\hline Adquisición de maquinaria y equipo & $-0,063$ & 0,034 & $-1,84$ & 0,072 \\
\hline Variable dicotómica de calidad & 1,950 & 1,074 & 1,82 & 0,075 \\
\hline Porcentaje de capacitación a empleados & 0,023 & 0,014 & 1,66 & 0,100 \\
\hline Nivel educativo medio & 0,189 & 0,099 & 1,91 & 0,061 \\
\hline Porcentaje de empleados & $-0,026$ & 0,012 & $-2,24$ & 0,029 \\
\hline Índice de normatividad laboral & $-1,657$ & 1,042 & $-1,59$ & 0,118 \\
\hline Constante & $-2,213$ & 1,794 & $-1,23$ & 0,223 \\
\hline Observaciones & 394 & & & \\
\hline Prob $>\mathrm{chi}^{2}$ & 0,00 & & & \\
\hline Seudo $\mathrm{R}^{2}$ & 0,28 & & & \\
\hline
\end{tabular}

Fuente: elaboración propia con datos de ENESTYC, 2001.

que la experiencia productiva de la empresa permite desarrollar capacidades para diseñar y aplicar mejores estrategias de capacitación.

Otras dos variables asociadas con una mayor probabilidad de que las empresas capaciten a su personal son la existencia de sindicato y el índice de actividades conjuntas con otras empresas (las cuales incluyen iniciativas conjuntas de capacitación), ambas significativas. Por último, la bondad de ajuste $(0,79)$ es elevada, considerando que se trata de un modelo de corte transversal y que el número de observaciones no es muy grande.

La segunda etapa de la estimación muestra los efectos de la capacitación y otras variables sobre la productividad (como variable sustitutiva de competitividad). En primer lugar, el coeficiente del efecto de la capacitación sobre la productividad multifactorial es significativo y positivo a un nivel de confianza del 90\% (cuadro 4). El ajuste por sesgo de selección - que corrige la endogeneidad de la variable de tratamiento (capacitación) - es negativo y significativo a un nivel de confianza de $90 \%$, lo cual corrobora que los errores de la ecuación de selección y la ecuación de resultados están correlacionados. Asimismo, las empresas que cuentan con certificados de calidad tienen en promedio una mayor productividad multifactorial que las que no los tienen. La industria electrónica en todo el mundo se caracteriza por tener altos estándares de calidad y es un elemento central de competitividad (cuadro 4).

La variable dicotómica de gasto en $\mathrm{I}+\mathrm{D}$ es significativa y positiva, es decir, tal actividad está asociada 
positivamente con la productividad multifactorial. Este resultado, además de reflejar las características tecnológicas y productivas de la industria electrónica mencionadas anteriormente, refleja en particular los esfuerzos internos de generación de nuevas tecnologías. Sin embargo, aunque la realización de actividades de I+D está asociada positivamente con la propensión a capacitar y con una mayor productividad multifactorial en la rama electrónica, se debe tener presente el tipo de $\mathrm{I}+\mathrm{D}$ que realiza esta rama industrial en México. Como se mencionó anteriormente, las mejoras de producto representan innovaciones principalmente a nivel nacional y un importante componente de los esfuerzos tecnológicos está enfocado a innovaciones de proceso.

La variable promedio de años de educación de la plantilla laboral es significativa y positiva. Este resultado — que se esperaría en cualquier industria y aún más en industrias que hacen uso intensivo de conocimientos - indica que la educación formal previa de los trabajadores es un factor importante de la productividad de las empresas.

\section{b) Rama automotriz (3841)}

La rama automotriz (cuadro 5) se caracteriza por efectuar un gasto medio en I+D (como porcentaje del valor agregado) y por hacer uso intensivo de capital. Entre las variables asociadas con incrementos en la probabilidad de que las empresas capaciten a sus trabajadores destacan la incorporación de nuevas tecnologías a través del uso y compra de patentes, y la introducción de maquinaria y equipo. De especial importancia es la innovación tecnológica a través de la mejora de procesos (mejoras a la maquinaria, a las líneas de producción, y otras).
El hecho de contar con certificados de calidad, y también la orientación exportadora (porcentaje de la producción exportada), están asociados significativa y positivamente con la probabilidad de que las empresas capaciten. Ls industria automotriz en México exhibe una elevada orientación exportadora y altos estándares de calidad, los cuales se extienden de las armadoras a todos sus proveedores. Estos dos factores van de la mano y requieren una capacitación continua de los trabajadores. Asimismo, las empresas que manufacturan productos o componentes para otras empresas (variable dicotómica de subcontratación) también tienen una propensión mayor a capacitar. Como se comentó antes, para ser proveedor en la industria automotriz se requieren certificaciones y fuertes controles de calidad, lo que puede explicar este último resultado. Finalmente, el nivel educativo promedio de la plantilla de trabajadores es una variable significativa y positiva, lo que indica que un mayor nivel educativo de la plantilla laboral está asociado con un incremento de las posibilidades de que las empresas capaciten. Este último resultado puede estar relacionado con el hecho de que la capacitación tiene una mayor rentabilidad si se ofrece a personas con mayor educación formal previa.

La segunda etapa de la estimación econométrica muestra que la capacitación en la empresa tiene un impacto positivo y significativo en su productividad (aunque este impacto es menor que en la rama electrónica). El ajuste por sesgo de selección es negativo y significativo (cuadro 6). El gasto en compra de patentes como porcentaje del valor agregado es significativo al 95\% de confianza y tiene un coeficiente positivo elevado, lo que refleja la importancia de este mecanismo

(Modelo probit de la capacitación en las empresas)

\begin{tabular}{|c|c|c|c|c|}
\hline & $\mathrm{dF} / \mathrm{dx}$ & Errores estimados robustos & $\mathrm{z}$ & $\mathrm{P}>|\mathrm{z}|$ \\
\hline Patentes & 0,036 & 0.018 & 2,03 & 0,042 \\
\hline Variable dicotómica de adquisición de maquinaria y equipo & 0,206 & 0,112 & 1,74 & 0,082 \\
\hline Variable dicotómica de mejora en procesos & 0,535 & 0,235 & 1,93 & 0,054 \\
\hline Variable dicotómica de calidad & 0,493 & 0,129 & 3,43 & 0,001 \\
\hline Producción exportada & 0,009 & 0,005 & 2,01 & 0,045 \\
\hline Nivel educativo medio & 0,068 & 0,021 & 3,39 & 0,001 \\
\hline Capital extranjero & 0,004 & 0,002 & 1,60 & 0,109 \\
\hline Variable dicotómica de subcontratación & 0,478 & 0,233 & 1,83 & 0,068 \\
\hline Observaciones & 1362 & & & \\
\hline Prob $>$ chi $^{2}$ & 0,000 & & & \\
\hline Seudo $\mathrm{R}^{2}$ & 0,520 & & & \\
\hline
\end{tabular}

Fuente: elaboración propia con datos de ENESTYC, 2001. 


\begin{tabular}{|c|c|c|c|c|}
\hline & Coeficiente & Errores estimados robustos & $\mathrm{z}$ & $\mathrm{P}>|\mathrm{z}|$ \\
\hline Variable dicotómica de capacitación & 1,147 & 0,627 & 1,83 & 0,068 \\
\hline Ajuste Heckman-Maddala-Lee & $-0,800$ & 0,409 & $-1,96$ & 0,052 \\
\hline Patentes & 7,637 & 3,350 & 2,28 & 0,024 \\
\hline Variable dicotómica de mejora de procesos & 0,513 & 0,296 & 1,73 & 0,085 \\
\hline Capacitación a obreros especializados & 0,002 & 0,004 & 0,65 & 0,513 \\
\hline Antigüedad media de trabajadores & 0,036 & 0,020 & 1,77 & 0,078 \\
\hline Tasa de empleados a obreros & 0,236 & 0,104 & 2,28 & 0,023 \\
\hline Capacidad instalada & 0,010 & 0,003 & 3,97 & 0,000 \\
\hline Variable dicotómica de calidad & 0,189 & 0,133 & 1,42 & 0,157 \\
\hline Variable dicotómica de capacitación externa & 0,002 & 0,001 & 1,84 & 0,067 \\
\hline Constante & $-1,350$ & 0,425 & $-3,18$ & 0,002 \\
\hline Observaciones & 1,362 & & & \\
\hline Prob $>\mathrm{chi}^{2}$ & 0,000 & & & \\
\hline Seudo $\mathrm{R}^{2}$ & 0,22 & & & \\
\hline
\end{tabular}

Fuente: elaboración propia con datos de ENESTYC, 2001.

en la incorporación de tecnología a esta rama y su impacto positivo en la productividad. La realización de actividades destinadas a la mejora de procesos está también asociada positivamente con el desempeño de la productividad.

La antigüedad media de los trabajadores también es un factor asociado positivamente con la productividad multifactorial en la industria automotriz. Es probable que una baja rotación de la mano de obra sea un incentivo para invertir en la formación de capital humano. Finalmente, la variable de capacitación externa es positiva y significativa. Dado que las principales fuentes de tecnología son externas a la empresa (patentes y compra de maquinaria y equipo), la capacitación externa por parte de los mismos proveedores de maquinaria u otras empresas y organizaciones está asociada positivamente con la productividad multifactorial.

\section{c) Rama de la confección de prendas de vestir (3220)}

Es importante señalar que para efectos de la estimación del modelo, en esta rama se excluyeron las microempresas debido a que la heterogeneidad de los datos dificultó el ajuste de la estimación. En particular, la varianza de variables como la capacitación y uso de tecnología fue muy elevada. ${ }^{17}$ En la rama de confección, la adquisición de maquinaria y equipo está asociada positivamente con la probabilidad de que una empresa

\footnotetext{
${ }^{17}$ Los establecimientos de tamaño micro y pequeño en esta rama tienen características que contrastan notablemente con las de los establecimientos de mayor tamaño.
}

capacite a sus trabajadores. Al igual que para las otras dos ramas, este resultado se asocia a la forma en que las empresas de la rama incorporan nuevas tecnologías. El control de calidad es también un elemento significativo ligado a incrementos de la probabilidad de que los establecimientos capaciten a sus trabajadores (cuadro 7).

A diferencia de los resultados para la rama automotriz, el nivel educativo medio de la plantilla laboral no es significativo para explicar si las empresas capacitan o no a su personal. Por otro lado, la antigüedad media de la plantilla laboral es significativa y negativa. Este resultado puede guardar relación con que la experiencia de los trabajadores disminuye las necesidades de capacitación y que gran parte de la capacitación es básica y solo busca que los nuevos obreros adquieran conocimientos sencillos para realizar sus funciones; no parece haber un esfuerzo continuo. Sólo el nivel educativo medio del personal directivo es significativo y positivo, lo cual indica que las capacidades gerenciales en estas empresas son un factor estratégico que puede orientar positivamente la organización de la producción y detectar oportunamente las necesidades de capacitación.

Los resultados de la segunda etapa de la estimación econométrica mostraron que la capacitación que imparten las empresas a sus trabajadores está asociada positivamente con su desempeño en materia de competitividad. Como se esperaba, el coeficiente es menor que en la rama electrónica. El ajuste por sesgo de selección fue significativo y negativo a un nivel de confianza de $90 \%$. Sin embargo, no fue posible encontrar más hallazgos consistentes. Otras variables asociadas al desempeño de la competitividad fueron contradictorias, debido a que 


\begin{tabular}{lrr}
\hline & $\mathrm{dF} / \mathrm{dx}$ & Errores estimados robustos \\
\hline Variable dicotómica de adquisición de maquinaria y equipo & 0,279 & 0,174 \\
Variable dicotómica de calidad & 0,429 & 0,136 \\
Porcentaje de obreros generales y especializados & 0,009 & 0,004 \\
Antigüedad media de los trabajadores & $-0,048$ & 0,023 \\
Nivel educativo medio de directivos & 0,034 & 0,013 \\
Indice de actividades conjuntas & 0,390 & 0,117 \\
\hline Observaciones & 2,98 & $-1,84$ \\
Prob $>$ chi $^{2}$ & 2215 & 3,02 \\
Seudo $\mathrm{R}^{2}$ & 0,000 & 0,024 \\
\hline
\end{tabular}

Fuente: elaboración propia con datos de ENESTYC, 2001.

las observaciones son altamente heterogéneas. En efecto, la varianza de los datos, sobre todo de las variables de uso y generación de tecnologías y de capacitación, no permitió obtener una estimación consistente. Incluso se realizó la estimación por conglomerados (por tamaños de empresa) y los resultados no fueron mejores.

\section{IV}

\section{Capacitación y competitividad en un entorno regional}

La sección III analizó el impacto de la capacitación en la competitividad de la empresa. Sin embargo, las empresas no son los únicos agentes en la economía que usan y generan nuevos conocimientos. Una gran variedad de organizaciones públicas y privadas — como universidades, colegios técnicos, cámaras empresariales y dependencias gubernamentales-incentivan y apoyan las actividades de capacitación en la empresa. Por otro lado, la capacitación en la empresa tiene impactos positivos en la competitividad de la industria y la economía local a través de la difusión de conocimientos, producto de la interacción entre las empresas y las organizaciones arriba mencionadas, así como a través de la movilidad del personal capacitado.

Con esto en mente, la presente sección analiza el impacto que tiene la capacitación en la competitividad de la economía local (nivel meso), y estudia el papel de las organizaciones públicas y privadas en la promoción y capacitación en la empresa. La hipótesis principal es que dicha capacitación, además de incrementar la competitividad de la empresa, tiene un impacto positivo en la competitividad de la región o localidad en donde opera. Bajo el enfoque evolucionista, la competitividad de una región, y en especial su capacidad de innovar, no recae exclusivamente en las empresas, sino también en diversas organizaciones públicas y privadas, y en la fortaleza de las relaciones entre ellas (Cooke, Gómez Uranga y Etxebarria, 1997; Howells, 1999; Carlsson, Jacobsson y otros, 2002; Iammarino, 2005). ${ }^{18}$

Para analizar el impacto de la capacitación en la competitividad a nivel meso, se realizó trabajo de campo en México en octubre de 2005. La investigación tiene un enfoque regional, es decir, el interés es conocer las organizaciones y las relaciones entre ellas en un espacio geográfico específico. Esta dimensión es de especial relevancia para este estudio, debido a la importancia de la cercanía geográfica para la interacción entre agentes y para la difusión de conocimientos (Cantwell y Iammarino, 2003; Cantwell y Molero, 2003; Malmberg, Sölvell y Zander, 1996).

\footnotetext{
${ }^{18}$ Este enfoque corresponde en particular a los sistemas regionales de innovación que en fechas recientes han adquirido particular relevancia.
} 
Como se mostró en la sección III, las características de la capacitación y su impacto en la competitividad varían de una industria a otra. De las tres ramas analizadas, la electrónica destaca por tener la mayor propensión a capacitar y el mayor rendimiento de la capacitación en términos de productividad. Asimismo, esta industria se caracteriza por su alto dinamismo tecnológico, que demanda un esfuerzo continuo de formación de capital humano. Por lo tanto, es un caso interesante para indagar en el aspecto central de este artículo: el impacto de la capacitación en la competitividad.

El trabajo de campo se concentró en la industria electrónica ubicada en Jalisco, México, la más importante del sector en el interior del país ${ }^{19}$ en términos de número de empresas, empleo generado y mayor captación de inversión extranjera directa. Jalisco también se presenta como un caso de estudio interesante en México debido a su desarrollo institucional relativamente mayor en materia de política industrial, así como por la fortaleza de su sistema de educación superior y técnica, y la mayor vinculación entre empresas, por un lado, y universidades, colegios técnicos y centros de investigación, por otro (Padilla, 2005).

El estudio de caso regional abarca una selección de organizaciones relacionadas con la capacitación en la empresa. ${ }^{20}$ Además de dos casos de estudio de empresas de la industria electrónica, se hicieron entrevistas en universidades y colegios técnicos, dependencias gubernamentales y organizaciones privadas.

En primer lugar, los casos de estudio de empresas muestran la importancia central de la capacitación para la competitividad de las empresas, en particular en la calidad, productividad e innovación, resultado que refuerza los hallazgos del análisis econométrico. La capacitación es una herramienta crucial para que los productos sean ensamblados y/o manufacturados de acuerdo con los más altos estándares de calidad, en una industria caracterizada por un gran dinamismo, alta competencia y estándares de calidad muy rigurosos. Asimismo, la inversión en capacitación se traduce en mayor productividad, asociada principalmente con menor retrabajo de productos y rechazos. Los estudios de caso indican que empresas que basan su competitividad en calidad e innovación dan un papel central a la capacitación de sus empleados. Esto se refleja en los recursos

\footnotetext{
${ }^{19}$ Estados fronterizos como Baja California y Chihuahua concentran un mayor número de empresas de la industria electrónica.

${ }^{20}$ La identificación de las instituciones se hizo sobre la base de la literatura especializada y de entrevistas con expertos en el tema (véase Padilla, 2005; Dussel, Palacios y Woo, 2003).
}

invertidos, en la profesionalización de los sistemas de detección de necesidades e impartición de capacitación, los mecanismos de evaluación, etc. ${ }^{21}$

En segundo lugar, la capacitación en la empresa en la industria electrónica de Jalisco es impartida y apoyada por organizaciones públicas y privadas. Tres tipos de organizaciones son de especial importancia: i) universidades y colegios técnicos; ii) dependencias gubernamentales, y iii) organizaciones privadas. Las primeras, además de formar recursos humanos a través de programas escolarizados de enseñanza, ofrecen cursos cortos a las empresas. Dichos cursos pueden ser de interés general para una industria o grupo de industrias y son impartidos por iniciativa propia de la organización académica, o responden a peticiones específicas de empresas y son diseñados de acuerdo con sus necesidades particulares.

Por su parte, el gobierno apoya la capacitación en la empresa a través de distintos mecanismos, como incentivos fiscales, fondos de apoyo a la capacitación, escuelas públicas de enseñanza técnica y superior, legislación y supervisión de su cumplimiento, y otros. Por último, las organizaciones privadas imparten cursos y prestan servicios de asistencia técnica orientados a identificar necesidades de capacitación y evaluar los resultados de la misma. Los cursos que imparten pueden ser de carácter general -como los relacionados con certificaciones, normalización, motivación y métodos de organización de la producción- o de carácter técnico y específicos para una empresa o grupo de empresas.

En tercer lugar, la capacitación impartida en las empresas puede tener efectos positivos en la competitividad de la región, principalmente a través de la difusión de conocimientos. El estudio de la industria electrónica en Jalisco permite identificar tres mecanismos importantes. El primero es el movimiento de personal entre empresas de la misma industria o de diferentes industrias. Cuando un ingeniero, técnico u obrero se cambia a una nueva empresa se lleva consigo la capacitación y habilidades obtenidas en el trabajo, las que pueden tener un impacto positivo en la competitividad de la nueva empresa que los contrata. Este personal capacitado también puede crear su propia empresa, lo que se conoce en la literatura especializada como spin-offs, usando los conocimientos adquiridos durante su experiencia laboral. El segundo mecanismo es la interacción de personal calificado de las empresas (ingenieros, técnicos, directivos) con

\footnotetext{
${ }^{21}$ Véase una descripción detallada de las actividades de capacitación de las empresas y su impacto en la competitividad en Padilla y Juárez (2006).
} 
universidades y colegios técnicos de la región. Además, los centros académicos suelen contratar profesores de media jornada que trabajan también en la industria. Estos profesores son capacitados y puestos al día en nuevas tecnologías, lo que les entrega conocimientos que posteriormente transmiten a sus alumnos. Los cursos que se imparten — pero también la asistencia técnica, los proyectos de investigación conjunta, las pasantías de profesores en empresas, entre otras cosas- ofrecen a las instituciones académicas nuevos conocimientos gracias a la interacción con personal de las empresas que ha sido previamente capacitado. El tercer mecanismo es la interacción del personal de las empresas con organizaciones privadas que les prestan servicios. La realización de proyectos conjuntos - por ejemplo, de capacitación y asistencia técnica- permite que los conocimientos impartidos al personal de las empresas se transfieran al personal de las organizaciones privadas de apoyo a la industria.

Los mecanismos descritos son de especial relevancia en industrias de alta tecnología en países en desarrollo,

\section{V}

\section{Conclusiones}

A nivel micro, el análisis econométrico permite concluir que la capacitación que las empresas imparten a sus trabajadores en las tres ramas estudiadas está asociada positivamente con la competitividad de esas empresas. La capacitación en la empresa se traduce en mayor competitividad debido a su impacto en la calidad de los productos, mayor eficiencia (menos trabajos rehechos y menos rechazos), mayor flexibilidad y mayor capacidad de innovar en productos y procesos.

Las variables asociadas a una mayor probabilidad de ofrecer capacitación en la empresa varían entre ramas industriales. Sin embargo, el control de calidad fue significativo y positivo en las tres ramas, lo que muestra por un lado la gran importancia de la capacitación para alcanzar altos estándares de calidad, y por otro, que los métodos de control de calidad demandan una política activa de capacitación. La capacitación está también fuertemente relacionada con la incorporación y generación de nuevas tecnologías. Los mecanismos varían entre las ramas examinadas: el gasto en I+D en la rama electrónica, la adquisición de maquinaria y equipo y compra de patentes en la rama automotriz y la adquisición de maquinaria y equipo en la rama de confección. como la electrónica en Jalisco. Estas industrias están comúnmente dominadas por empresas transnacionales que operan con tecnología de punta o con tecnología más avanzada que la existente en empresas nacionales, así como en universidades, colegios técnicos y organizaciones privadas de apoyo al sector empresarial. En consecuencia, los conocimientos que primero son transferidos al personal de empresas transnacionales, a través de capacitación formal e informal, pueden convertirse en una valiosa fuente de actualización para el resto de la industria y, en general, para la economía local. En efecto, el personal capacitado es un agente de diseminación a la economía receptora de los conocimientos transferidos por empresas transnacionales. No obstante, cabe reconocer que estos derrames no son un resultado inmediato y directo. La evidencia empírica de diversos países en desarrollo muestra que las empresas transnacionales pueden operar en condiciones de enclave, con escasa vinculación con la economía local y con características productivas y tecnológicas muy básicas, limitando significativamente los derrames al resto de la región.
La predicción central del modelo econométrico es que la capacitación tiene un efecto positivo en la competitividad de la empresa, y que la magnitud del impacto es mayor en industrias caracterizadas por un mayor dinamismo tecnológico. Las ramas industriales que hacen uso de nuevas tecnologías tanto de proceso como de producto, y que también las generan, tienen que invertir en capacitación que les permita asimilar e incluso crear nuevas tecnologías. Esta relación puede generar un círculo virtuoso: el cambio tecnológico requiere capacitación y esta es esencial para la innovación. En este sentido, la capacitación está ligada a un proceso activo de uso, mejora y generación de conocimientos.

La competitividad a nivel de empresa también está asociada con diversas variables, dependiendo de las características productivas y tecnológicas propias de la rama industrial a la que pertenece. En la rama electrónica, el gasto en I+D, que es una fuente interna de generación de conocimientos, y también el control de calidad están asociados positivamente con la competitividad. Como se señaló anteriormente, la interpretación de este resultado tiene que tomar en cuenta también el tipo de actividades de I+D que llevan a cabo las empresas electrónicas en 
México. Por otro lado, en la rama automotriz la capacitación de obreros especializados es lo que mayor impacto tiene en la competitividad de la empresa, a la que también contribuyen la compra de patentes y la presencia de métodos de control de calidad. En la rama de la confección no fue posible obtener estimaciones consistentes, debido a la gran varianza observada en las variables independientes.

La segmentación por contenido tecnológico resultó útil para identificar diferencias en la estrategia de capacitación en la empresa y su impacto en la competitividad de la industria. Sin embargo, es importante reconocer que esta no es la única segmentación relevante para analizar el impacto de la capacitación en la competitividad. Dos líneas futuras de investigación se desprenden de esta investigación: a) las empresas de menor tamaño tienen una menor propensión a capacitar y el análisis de las limitaciones que estas enfrentan para contar con una estrategia activa de capacitación de acuerdo a sus necesidades; b) el análisis del impacto que tiene la posición en la cadena de valor (diseño, I+D, ensamble, manufactura, comercialización, etc.) en las decisiones y los recursos disponibles para ofrecer capacitación a los empleados. Por otro lado, la incorporación de datos de panel sería una extensión al modelo econométrico aquí presentado, la cual podría arrojar resultados interesantes de la evolución del impacto de la capacitación en la competitividad.

A nivel meso, el estudio de caso de la industria electrónica en una región de México también permite concluir que la capacitación en la empresa tiene un impacto positivo en la competitividad de la región. La difusión al resto de la región de los conocimientos tecnológicos que inicialmente se transmitieron al personal de las empresas, es claramente un beneficio social. El movimiento del personal; la vinculación entre empresas y con universidades y colegios técnicos, y la contratación de servicios de capacitación de empresas privadas son algunos de los mecanismos para difundir, perfeccionar y generar nuevos conocimientos, lo que puede contribuir en gran medida a elevar la competitividad de la región.
De esta manera, una estrategia conjunta de capacitación, basada en alianzas o cooperación entre empresas y organizaciones públicas, puede tener efectos muy positivos a nivel macro y meso. Por un lado, refuerza la estrategia de capacitación de las empresas; por otro, fortalece las capacidades regionales que permiten tener una industria manufacturera local más competitiva, y a la vez hace que la región pueda atraer nuevas y mejores inversiones.

El sector público en particular puede desempeñar un papel central al incentivar y facilitar las actividades de capacitación en la empresa y la difusión de conocimientos. Los hallazgos del presente trabajo sugieren que las iniciativas públicas dirigidas a incentivar o a apoyar directamente la capacitación en la empresa deben tomar en cuenta las necesidades específicas de cada industria, es decir, sus características productivas y tecnológicas. Asimismo, las políticas públicas en materia de capacitación deben diseñarse dentro de un marco integral y regional que, reconociendo las particularidades y necesidades del entorno local, incorpore la importancia de integrar los esfuerzos de instituciones como universidades, colegios técnicos, organizaciones privadas de asistencia técnica y cámaras empresariales.

La oferta de formación de capital humano tendrá que actualizarse y transformarse continuamente ante el rápido cambio tecnológico, acentuado por la expansión y penetración de las tecnologías de la información. La capacitación en la empresa, por su naturaleza, es más flexible para incorporar nuevos conocimientos y habilidades. La formación profesional, incluida la capacitación en la empresa, debe hacer uso de nuevos métodos de enseñanza - como el desarrollo de la autonomía y creatividad del individuo - que respondan al rápido cambio tecnológico y faciliten el aprendizaje y el desarrollo continuo de habilidades (Rolf, 2002).

Por último, dada la estrecha relación entre capacitación, innovación y calidad, las políticas públicas de apoyo a la capacitación deben diseñarse de manera conjunta con las políticas de fomento de la innovación y la calidad. La visión integral del fenómeno permitirá hacer un mejor uso de los recursos y obtener mejores resultados. 
APÉNDICE A

Lista de variables

Variables

Productividad total de los factores (índice)

Variable dicotómica de capacitación

Variable dicotómica de la rama de confección

Variable dicotómica de la rama electrónica

Variable dicotómica de la rama automotriz

Tamaño

\section{Capital extranjero}

Variable dicotómica de sindicato

Variable dicotómica de subcontratación

Relación de empleados a obreros

Porcentaje de directores

Porcentaje de empleados

Porcentaje de obreros especializados

Porcentaje de obreros generales

Capacidad instalada

Antigüedad de la empresa

Producción exportada

Variable dicotómica de calidad

Índice de actividades conjuntas

Índice de normatividad laboral

Nivel educativo medio

Antigüedad media de trabajadores

Porcentaje de capacitación por categoría ocupacional

Variable dicotómica de capacitación interna formal

Variable dicotómica de capacitación interna informal

Variable dicotómica de capacitación externa

Adquisición de maquinaria y equipo

Variable dicotómica de adquisición de maquinaria y equipo

Compra de patentes

Patentes

Ingeniería básica

Investigación y desarrollo (I+D)

Variación dicotómica de mejora de procesos

Variación dicotómica de I+D
Construcción de variables

Cociente del valor agregado y el valor de los factores capital y trabajo empleados en la producción para el año 2000

1 si la empresa se capacitó y 0 en otro caso

1 cuando la empresa pertenece a la rama de confección y 0 en otro caso 1 cuando la empresa pertenece a la rama de electrónica y 0 en otro caso 1 cuando la empresa pertenece a la rama de automotriz y 0 en otro caso

Variable con cuatro posibles valores: 1 si laboran menos de 16 empleados; 2 , si laboran entre 16 y 100; 3 si laboran entre 101 y 250; y 4 si laboran más de 250.

Porcentaje de capital social extranjero de la empresa

1 ante la existencia de un sindicato y 0 en otro caso

1 si la empresa realizó actividades de subcontratación y 0 en otro caso

Cociente entre el número de empleados y obreros

Porcentaje de trabajadores con una categoría ocupacional asociada a actividades directivas

Porcentaje de trabajadores con una categoría ocupacional de empleados (incluye a profesionistas, técnicos, empleados administrativos y supervisores)

Porcentaje de trabajadores con una categoría ocupacional independiente que dominan ampliamente su actividad

Porcentaje de los trabajadores de la plantilla laboral que tienen una categoría ocupacional de obreros generales

Porcentaje de utilización de la capacidad productiva de los establecimientos

Años de operación del establecimiento

Porcentaje de la producción que se exporta

1 si la empresa cuenta con control de calidad y 0 en otro caso

Índice que indica la proporción de actividades conjuntas que realiza el establecimiento con otras empresas: ventas, compra de materias primas, acceso a crédito, capacitación, I+D, utilización de maquinaria y equipo adquisición de maquinaria y equipo

Índice que resume si la empresa cuenta con: funciones de categorías salariales, rotación de personal, contratación de personal eventual, trabajo subcontratado, creación de puestos de confianza, recorte de personal, selección de personal y promoción de personal

Promedio de años de educación de la plantilla laboral total

Promedio de años de antigüedad de la plantilla laboral

Porcentaje de la capacitación horas-hombre impartida a los directores, empleados, obreros especializados u obreros generales

1 si la empresa dio capacitación por un compañero y 0 en otro caso

1 si la empresa dio capacitación por un agente instructor y 0 en otro caso

1 si la empresa dio capacitación a través de un agente externo y 0 en otro caso

Gasto en adquisición de maquinaria y equipo en el año 2000, como porcentaje del valor agregado

1 si la empresa adquirió maquinaria y equipo en el 2000 y 0 en otro caso

Gasto en compra de patentes en el 2000, como porcentaje del valor agregado

Gasto en uso de patentes en el 2000, como porcentaje del valor agregado

Gasto en ingeniería básica en el 2000, como porcentaje del valor agregado

Gasto en I+D en el 2000, como porcentaje del valor agregado

1 si la empresa llevó cabo mejora de procesos en el 2000 y 0 en otro caso

1 si la empresa realizó I+D en el 2000 y 0 en otro caso 


\section{Bibliografía}

Abdel, G. (2004): El sector autopartes en México, México, D.F., Centro de Estudios de Competitividad, Instituto Tecnológico Autónomo de México (ITAM).

Altenburg, T., W. Hillebrand y J. Meyer-Stamer (1998): Building Systemic Competitiveness: Concept and Case Studies from Mexico, Brazil, Paraguay, Korea and Thailand, Reports and Working Papers, N 3/1998, Berlín, Instituto Alemán de Desarrollo (IAD).

Barnow, B.S., G. Cain y A. S. Goldberger (1981): Issues in the analysis of selection bias, en W.E. Stromsdorfer y G. Farkas (comps.), Evaluation Studies Review Annual, vol. 5, N ${ }^{\circ} 3$, Beverly Hills, Sage.

Bartel, A. (1989): Formal Employee Training Programs and Their Impact on Labor Productivity: Evidence from a Human Resources Survey, NBER Working Papers, No 3026, Cambridge, Massachusetts, National Bureau of Economic Research.

Batra, G. y H.W. Tan (2002): Upgrading work force skills to create high-performing firms, en I. Nabi y M. Luthria (comps.), Building Competitive Firms, Washington, D.C., Banco Mundial.

Becker, G. (1964): Human Capital, Cambridge, Massachusetts, National Bureau of Economic Research.

BID (Banco Interamericano de Desarrollo) (2004): Competitividad: conceptos y buenas prácticas, Washington, D.C.

Booth, A. y D. Snower (1996): Acquiring Skills: Market Failures, Their Symptoms and Policy Responses, Cambridge, Cambridge University Press.

Cantwell, J. y J. Molero (2003): Introduction, en J. Cantwell y J. Molero (comps.), Multinational Enterprises, Innovative Strategies and Systems of Innovation, Londres, Edward Elgar.

Cantwell, J. y S. Iammarino (2003): Multinational Corporations and European Regional Systems of Innovation, Londres, Routledge.

Carlsson, B., S. Jacobsson y otros (2002): Innovation systems: analytical and methodological issues, Research Policy, vol. 31, $\mathrm{N}^{\mathrm{o}}$ 2, Amsterdam, Elsevier.

Cohen, W., A. Goto y otros (2002): R\&D spillovers, patents and the incentives to innovate in Japan and the United States, Research Policy, vol. 31, No 8-9, Amsterdam, Elsevier, diciembre.

CONACYT (Consejo Nacional de Ciencia y Tecnología) (2003): Informe General del Estado de la Ciencia y la Tecnología 2003, México, D.F.

Cooke, P., M. Gómez Uranga y G. Etxebarria (1997): Regional innovation systems: institutional and organisational dimensions, Research Policy, vol. 26, No 4-5, Amsterdam, Elsevier, diciembre.

Dussel, E., J.J. Palacios y G. Woo (coords.) (2003): La industria electrónica en México: problemática, perspectiva y propuestas, Jalisco, México, Universidad de Guadalajara.

Gallart, M.A. (2001): La articulación entre el sector público y la empresa privada en la formación profesional de América Latina, en G. Labarca (coord.), Formación para el trabajo: ¿pública o privada?, Montevideo, Organización Internacional del Trabajo (OIT)/Centro Interamericano de Investigación y Documentación sobre Formación Profesional (CINTERFOR).

Giuliani, E., C. Pietrobelli y R. Rabelotti (2005): Upgrading in global value chains: lessons from Latin American clusters, World Development, vol. 33, N 4, Amsterdam, Elsevier.

Griliches, Z. (1967): Production functions in manufacturing: some preliminary results, en M. Brown (comp.), The Theory and Empirical Analysis of Production, Nueva York, Columbia University Press.

Howells, J. (1999): Regional systems of innovation?, en D. Archibugi, J. Howells y J. Michie, Innovation Policy in a Global Economy, Cambridge, Cambridge University Press.

Iammarino, S. (2005): An evolutionary integrated view of regional systems of innovation. Concepts, measures and historical perspectives, European Planning Studies, vol. 13, No 4, Londres, Taylor and Francis.

Lara Rivero, A. y A. Díaz-Berrio (2003): Cambio tecnológico y socialización del conocimiento tácito, Comercio exterior, vol. 53, México, D.F., Banco Nacional de Comercio Exterior (BANCOMEXT).

Lynch, L. y S. Black (1995): Beyond the Incidence of Training: Evidence from a National Employers Survey, NBER Working Papers, No 5231, Cambridge, Massachusetts.

Malmberg, A., O. Sölvell e I. Zander (1996): Spatial clistering, local accumulation of knowledge and firm competitiveness, Geografiska Annaler, vol. 78, N² 2, Oxford, Reino Unido, Blackwell Publishing.

McFetridge, D. (1995): Competitiveness: Concepts and Measures, Occasional Paper, No 5 , Ottawa, Industry Canada.

Mincer, J. (1981): Human Capital and Economic Growth, NBER Working Papers, $N^{\circ} 803$, Cambridge, Massachusetts, National Bureau of Economic Research.

(1994): Investment in U.S. Education and Training, NBER Working Papers, No 4844, Cambridge, Massachusetts, National Bureau of Economic Research.

Nabi, I. y M. Luthria (comps.) (2002): Building Competitive Firms, Washington, D.C., Banco Mundial.

OCDE (Organización de Cooperación y Desarrollo Económicos) (2001): Measuring Productivity. OECD Manual, París.

(2004a): A New World Map in Textiles and Clothing, París.

(2004b): Science, Technology and Industry Outlook 2004, París.

Olley, S. y A. Pakes (1996): The dynamics of productivity in telecommunications equipment industry, Econometrica, vol. 64, $\mathrm{N}^{\mathrm{o}} 6$, Nueva York, Econometric Society.

Padilla, R. (2005): La industria electrónica en México, México, D.F., Centro de Estudios de Competitividad, Instituto Tecnológico Autónomo de México (ITAM).

Padilla, R. y M. Juárez (2006): Efectos de la capacitación en la competitividad de la industria manufacturera, serie Estudios y perspectivas, Nº 49, LC/L.2536-P, México, D.F., Sede Subregional de la CEPAL en México. Publicación de las Naciones Unidas, $\mathrm{N}^{\mathrm{o}}$ de venta: S.06.II.G.63.

Pavitt, K. (1984): Sectoral patterns of technical change: towards a taxonomy and a theory, Research Policy, vol. 13, N $^{\circ} 6$, Amsterdam, Elsevier, diciembre.

Pavcnik, N. (2002): Trade, liberalization, exit and productivity improvements: evidence from Chilean plants, Review of Economic Studies, vol. 69, № 1, Oxford, Reino Unido, Institute of Economics and Statistics, University of Oxford.

Porter, M. (1985): Competitive Advantage, Nueva York, The Free Press.

Rolf, A. (2002): Formación profesional: nuevas tendencias y perspectivas, Montevideo, Organización Internacional del Trabajo (OIT)/Centro Interamericano de Investigación y Documentación sobre Formación Profesional (CINTERFOR).

Romer, P. (1989): Human Capital and Growth: Theory and Evidence, NBER Working Papers, No 3173 , Cambridge, Massachusetts, National Bureau of Economic Research.

Spencer, M. y H. Hazard (1988): International Competitiveness, Cambridge, Massachusetts, Ballinger.

Tan, H.W. y G. Batra (1995): Enterprise Training in Developing Countries, PSD Occasional Paper, $\mathrm{N}^{\circ}$ 9, Washington, D.C., Banco Mundial.

Tan, H.W. y G. López-Acevedo (2003): In-firm Training for the Knowledge Economy, Policy Research Working Paper, № 2957, Washington, D.C., Banco Mundial. 